\title{
Clinical value of radiomics and machine learning in breast ultrasound: a multicenter study for differential diagnosis of benign and malignant lesions
}

\author{
Valeria Romeo ${ }^{1} \cdot$ Renato Cuocolo $^{2,3} \cdot$ Roberta Apolito $^{1} \cdot$ Arnaldo Stanzione $^{1}$ (D) - Antonio Ventimiglia ${ }^{1}$. \\ Annalisa Vitale ${ }^{1}$. Francesco Verde ${ }^{1} \cdot$ Antonello Accurso $^{1} \cdot$ Michele Amitrano ${ }^{1} \cdot$ Luigi Insabato $^{1} \cdot$ Annarita Gencarelli $^{1}$. \\ Roberta Buonocore ${ }^{4}$. Maria Rosaria Argenzio ${ }^{4}$. Anna Maria Cascone ${ }^{4}$. Massimo Imbriaco ${ }^{1}$. Simone Maurea ${ }^{1}$. \\ Arturo Brunetti ${ }^{1}$
}

Received: 18 November 2020 / Revised: 6 April 2021 / Accepted: 22 April 2021 / Published online: 21 May 2021

(C) The Author(s) 2021

\begin{abstract} radiologist's performance is improved by using ML. of a radiologist without and with the aid of ML, using McNemar's test. performance improved when using ML $(80.2 \%)$, but not significantly $(p=0.508)$. lesions on ultrasound images.

Key Points

- Machine learning showed good accuracy in discriminating benign from malignant breast lesions

- The machine learning classifier's performance was comparable to that of a breast radiologist

- The radiologist's accuracy improved with machine learning, but not significantly
\end{abstract}

Objectives We aimed to assess the performance of radiomics and machine learning (ML) for classification of non-cystic benign and malignant breast lesions on ultrasound images, compare ML's accuracy with that of a breast radiologist, and verify if the

Methods Our retrospective study included patients from two institutions. A total of 135 lesions from Institution 1 were used to train and test the ML model with cross-validation. Radiomic features were extracted from manually annotated images and underwent a multistep feature selection process. Not reproducible, low variance, and highly intercorrelated features were removed from the dataset. Then, 66 lesions from Institution 2 were used as an external test set for ML and to assess the performance

Results After feature selection, 10 of the 520 features extracted were employed to train a random forest algorithm. Its accuracy in the training set was $82 \%$ (standard deviation, $\mathrm{SD}, \pm 6 \%$ ), with an AUC of 0.90 (SD \pm 0.06 ), while the performance on the test set was $82 \%(95 \%$ confidence intervals $(\mathrm{CI})=70-90 \%)$ with an AUC of $0.82(95 \% \mathrm{CI}=0.70-0.93)$. It resulted in being significantly better than the baseline reference $(p=0.0098)$, but not different from the radiologist $(79.4 \%, p=0.815)$. The radiologist's

Conclusions A radiomic analysis combined with ML showed promising results to differentiate benign from malignant breast

Keywords Machine learning · Breast cancer · Ultrasound

Valeria Romeo and Renato Cuocolo contributed equally to the study and should be considered co-first authors.

Arnaldo Stanzione

arnaldo.stanzione@unina.it

1 Department of Advanced Biomedical Sciences, University of Naples "Federico II", Via S. Pansini, 5, 80131 Naples, Italy

2 Department of Clinical Medicine and Surgery, University of Naples "Federico II", Naples, Italy
3 Laboratory of Augmented Reality for Health Monitoring (ARHeMLab), Department of Electrical Engineering and Information Technology, University of Naples "Federico II", Naples, Italy

4 Department of Radiology, A.O.U. San Giovanni di Dio e Ruggi d'Aragona, Salerno, Italy 


\begin{tabular}{ll}
\multicolumn{2}{l}{ Abbreviations } \\
AI & Artificial intelligence \\
BI-RADS & Breast Imaging-Reporting and Data System \\
GLCM & Gray level co-occurrence matrix \\
ICC & Intraclass correlation coefficient \\
ML & Machine learning \\
NIR & No information rate \\
NPV & Negative predictive value \\
PPV & Positive predictive value \\
RF & Forest Ensemble Algorithm \\
RFECV & Cross Validated Recursive Feature Elimination \\
ROIs & Regions of interest \\
SD & Standard deviation \\
SMOTE & Synthetic Minority Oversampling Technique \\
US & Ultrasound
\end{tabular}

\section{Introduction}

Ultrasound (US) has gained an established role in the assessment of breast lesions, showing several indications in female subjects including cases of palpable lumps, as first diagnostic tool in patients younger than 40, and for the evaluation of suspicious findings at mammography or magnetic resonance imaging [1]. According to the Breast Imaging-Reporting and Data System (BI-RADS) risk assessment and quality assurance tool, lesions are classified into different categories reflecting malignancy probability [2]. Several BI-RADS US descriptors are provided to aid in standardizing breast lesion characterization. According to a recent meta-analysis, the pooled sensitivity and specificity of US used as primary tool in detecting breast cancer lesions are $80.1 \%$ and $88.4 \%$, respectively [3].

Radiomics is a complex multi-step process that allows extracting quantitative data from medical images, for example using texture analysis, to build clinically useful prediction models and decision support tools $[4,5]$. In oncologic patients, radiomics features can be used to non-invasively assess intratumoral heterogeneity on routinely performed imaging exams [6]. When applied to medical images, artificial intelligence (AI) techniques employing machine learning (ML) algorithms have shown valuable results in image-recognition tasks, also being able to extract quantitative parameters reflecting image heterogeneity [7]. Mainly embraced for classification tasks, different ML approaches can be considered, with the most commonly applied in radiology being supervised learning (requiring labeled input data) and unsupervised learning (requiring unlabeled input data) [8]. Furthermore, several different algorithms are currently available, from those more easily interpretable (such as decision trees) to more complicated and harder to interpret ones (such as the convolutional neural networks used in deep learning) [9]. A large variety of possible clinical applications for $\mathrm{AI}$ in breast imaging has been described, applied to either US, digital breast tomosynthesis, or magnetic resonance imaging, ranging from differential diagnosis of breast lesions to breast cancer molecular subtype identification and prognosis prediction [10, 11]. According to previous experiences, radiomic analyses applied to breast US images have shown a good accuracy in the differential diagnosis of BI-RADS 4 and 5 lesions as well as to discriminate triple negative breast cancer from fibroadenomas $[12,13]$. Often, these investigations lack external validation as analyzed data originates from a single institution. Similarly, the added clinical value of the proposed ML tools is not always assessed, leaving some doubts on the real-world benefits of AI.

Therefore, the scope of this study was threefold: (1) to assess the accuracy of a radiomic approach paired to ML applied to US images acquired in routine clinical practice to differentiate benign from malignant BIRADS $2-5$ breast lesions, with internal and external testing; (2) to compare its diagnostic accuracy with that of a dedicated breast radiologist; (3) to verify whether the performance of the radiologist could be improved by the use of the proposed ML algorithm.

\section{Materials and methods}

\section{Patient population}

The institutional review board approved this retrospective study and written informed consent was waived. All breast ultrasound examinations performed between November 2018 and June 2019 at the University Hospital "San Giovanni di Dio e Ruggi D'Aragona" in Salerno, Italy (Institution 1), and the Diagnostic Imaging Unit of the University of Naples "Federico II," Italy (Institution 2). Clinical indications for performing breast US were both routine check-up and assessment of palpable breast lesions or diagnostic in-depth analysis of breast lesions detected elsewhere. US examinations were performed by two radiologists in each institution, with 8 to 20 years of experience in breast imaging. Inclusion criteria were as follows: $>18$-year-old patients with at least one BI-RADS 2, 3, 4, or 5 lesion. Exclusion criteria were as follows: unavailable follow-up for BI-RADS 3 lesions, or pathological confirmation for BI-RADS 4 and 5 lesions; US images not suitable for radiomic analysis due to artifacts; BI-RADS 2 cystic lesions. Breast lesions from Institution 1 were used as the training set, while those from Institution 2 were used as an external test set, to assess the radiologist's performance with and without the aid of AI. The standard of reference consisted of 6 months follow-up (US and/or mammography) for BI-RADS 3 lesions and pathology 
examination by means of Tru-Cut biopsy or surgical excision for BI-RADS 4 and 5 lesions.

\section{Image acquisition}

US examinations were performed using a LOGIQ S8, GE Healthcare (Institution 1) and a Logos HiVision E-Hitachi (Institution 2) US scanners, employing a high-frequency linear probe with radial, transverse, and longitudinal scans on both breasts. DICOM images were recorded and stored in the respective institutional digital archives.

\section{Image conversion and segmentation}

US examinations were evaluated by a dedicated breast radiologist (VR) with 8 years of experience in breast imaging who selected and retrieved the DICOM image of each breast lesion. 2D B-mode images in which the lesion was fully included, free from artifact and any measurements, were selected. As US images were originally encoded as three-channel RGB images, they were converted to grayscale applying an ITU-R 601-2 luma transform:

$\mathrm{L}=\mathrm{R} * 299 / 1000+\mathrm{G} * 587 / 1000+\mathrm{B} * 114 / 1000$

Where $\mathrm{L}$ is the luminance value, and $\mathrm{R}, \mathrm{G}$, and $\mathrm{B}$ the original values for the red, green, and blue channels for each pixel. This was performed with the Image module of the PILLOW Python package (v2.2.1).
Subsequently, the same radiologist performed manual lesion segmentation using a dedicated software (ITKSNAP, v3.8.0) obtaining 2D regions of interest (ROIs) (Fig. 1) [14]. To assess feature stability, manual segmentation was also performed independently by two senior radiology residents (A.V. and A.V.) on 30 randomly selected patients from the training set in order to calculate feature intraclass correlation coefficient (ICC) [15].

\section{Image preprocessing and feature extraction}

A dedicated open-source Python-based software (PyRadiomics, v2.2.0) was employed for image preprocessing and 2D radiomic feature extraction [16] [17].

As voxels were already isotropic in-plane, no resampling was necessary prior to feature extraction. On the other hand, gray-level whole-image normalization was performed to ensure comparability of images acquired on different scanners and with varying settings, with a resulting range of $0-600$. As suggested by the developers, a fixed bin width $(=3)$ was used for discretization. Other than from the original images, features were also extracted from Laplacian of Gaussian (LoG, sigma $=1,2,3,4,5$ ) and wavelet (all high- and low-pass filter combinations on $\mathrm{x}$ and $\mathrm{y}$ planes) filtered ones. The use of image filters can reduce image noise and highlight textural characteristics. In particular, the LoG filter performs an image smoothing operation, enhancing structural edges within the image of interest. In this setting, the sigma value specifies the desired fineness or coarseness of the resulting output
Fig. 1 Examples of lesion annotation. The upper row $(\mathbf{a}, \mathbf{b})$ shows placement of a region of interest on a benign lesion, while $\mathbf{c}$ and $\mathbf{d}$ depict a malignant lesion before and after manual segmentation.

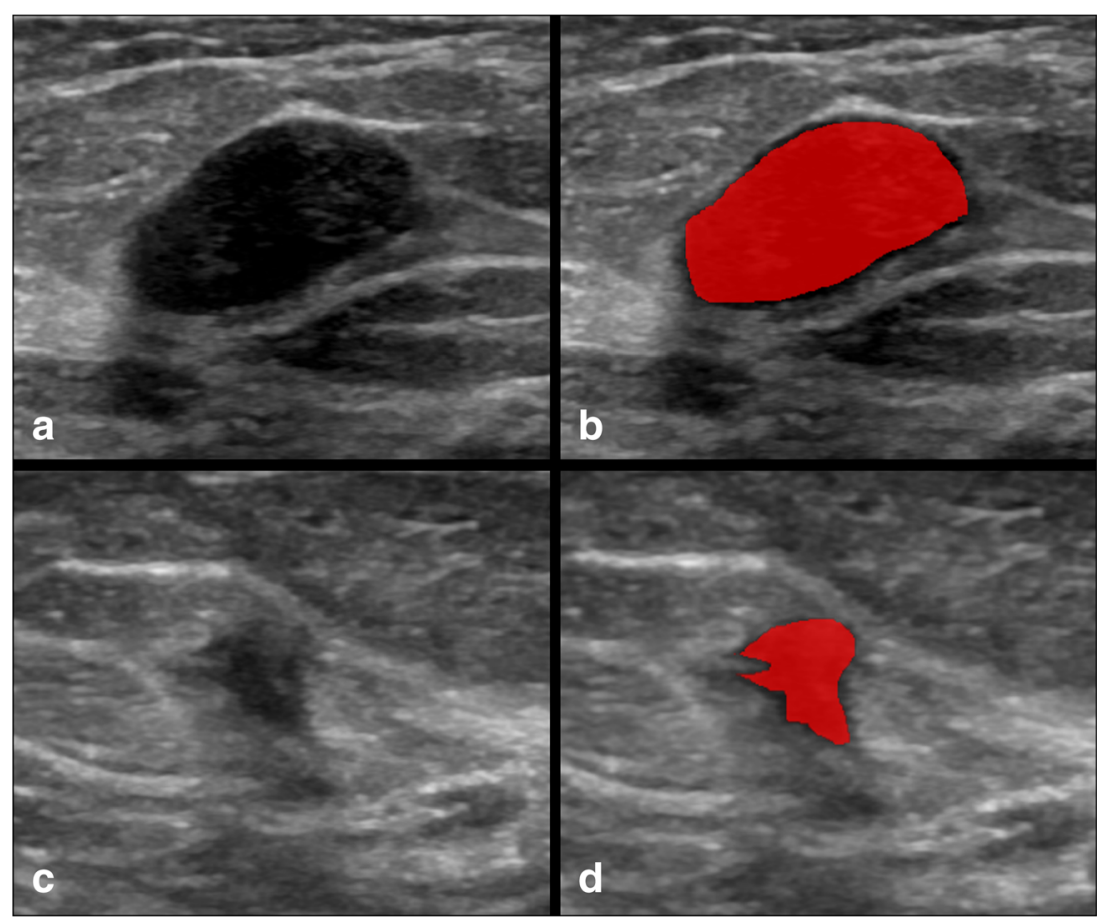


(lower values produce finer images and vice versa) [18]. Wavelet decompositions represent an alternative approach to remove low signal areas from the images (i.e., image smoothing and edge detection). Using high- and low-pass filter combinations, the original image is decomposed in distinct components, expanding the original signal [19]. As the best practice for medical image analysis is not established, these alternative filtering approaches have been both included in the investigation.

In regard to feature classes, 2D shape, first order, gray level co-occurrence matrix (GLCM), gray level run length matrix, gray level size zone matrix, and gray level dependence matrix ones were extracted. In particular, as image acquisition could vary in terms of depth and zoom, only adimensional 2D shape features were included to avoid biases, in particular perimetersurface ratio, sphericity, spherical disproportion, and elongation. For the remaining classes, all available features were calculated with the exception of GLCM sum average, as suggested by the PyRadiomics developers due to known redundancy with other GLCM parameters.

Formulas and definitions of the extracted features can be found on the official documentation (https://pyradiomics. readthedocs.io/en/latest/features.html).

\section{Data analysis and feature selection}

Feature stability testing was performed by calculating a twoway random effect, single rater, absolute agreement ICC for each. Only features with good reproducibility (ICC value $\geq$ 0.75 ) were considered stable and included in the following steps $[15,20]$. ICC calculation was performed using the $\mathrm{R}$ "irr" package [21]. The numbers of patients $(n=30)$ and readers $(n=3)$ as well as the ICC cutoff value were based on suggestions by recent guidelines and previous ML studies $[15,20]$. A MinMax scaler with $0-1$ range was fitted on the training data alone, to avoid any information leakage, and used to transform both training and test sets. Successively, non-informative features showing low variance $(\leq 0.01)$ were excluded. Similarly, highly intercorrelated features were discarded based on the pairwise correlation matrix $(r \geq 0.8)$. At this point, the Synthetic Minority Over-sampling Technique (SMOTE) was employed on the training data to balance the dataset $[22,23]$. In detail, SMOTE creates new instances (i.e., synthetic patients) of the minority class by interpolation of data from $k$ ( $=5$ in our study) nearest neighbors from the original population with the same label. The process is repeated until the two classes are perfectly balanced. Finally, stratified 10-fold cross-validated recursive feature elimination (RFECV) with a Logistic Regression (LBFGS solver) estimator identified the optimal number of parameters to train the ML algorithm. These data processing steps were conducted using the pandas and scikit-learn Python packages $[21,24]$.

\section{Machine learning analysis}

Given the tabular nature of the data, expected number of instances available, and previous experiences, also following the recommendations made by the scikit-learn developers, a Random Forest (RF) ensemble algorithm was selected for this classification task.

Algorithm performance during the random search tuning process was assessed on the training set through 5-fold stratified cross-validation. This approach is more robust than a single train-test split and can be expected to give a better estimation of generalizability [25]. In stratified cross-validation, each of the folds the data is split preserves the class balance and is used as a validation set for an algorithm trained on the remaining $(n=4)$ data folds. Then, the final model was fitted on the whole training set and tested on the data from Institution 2. Its accuracy was also compared to a baseline reference value (no information rate, NIR) corresponding to the accuracy obtainable by classifying all lesions as belonging to the most frequent class (i.e., the mode of the classes). A $p$ value $\leq 0.05$ was considered statistically significant. The Brier score was calculated for the model on the test set, as well as a calibration curve, to assess prediction and calibration loss of predicted probability and lesion class.

The machine learning analysis was performed using the scikit-learn Python package. Accuracy metrics were computed with the same Python package and the caret R package [21, 24].

\section{Radiological evaluation}

A dedicated breast radiologist (R.B., 8 years of experience) from Institution 1 evaluated the same US images of the test set used for the ML analysis and classified each lesion as benign or malignant, according to the BI-RADS V edition. In detail, the radiologist assessed lesion shape, margins, orientation, echo-pattern, and posterior features assigning a score from 2 to 5 . BI-RADS scores were then dichotomized as $2-3=$ benign, and 4-5 = malignant for the subsequent analysis. The radiologist was blinded to patient clinical history and final diagnosis. After a 4-week washout period, the same radiologist performed a new evaluation, this time with the availability of ML predictions and probabilities for each lesion. The accuracy of the radiologist was calculated using the caret $\mathrm{R}$ package and also compared to the NIR baseline accuracy reference.

\section{Statistical analysis}

Kolmogorov-Smirnov test was first performed to assess whether data were normally distributed. Accordingly, t-test and Mann-Whitney U test were performed to assess differences in terms of age and lesion size (maximum diameter) of 
malignant and benign breast lesions between training and test sets. Accuracy, sensitivity, specificity, and positive and negative predictive values of both ML classifier and expert radiologist were calculated. McNemar's test was performed to assess differences in the performance between ML and the human reader and between the reader without and with the use of ML. A $p$ value $\leq 0.05$ was considered statistically significant.

\section{Results}

\section{Patient population}

Based on inclusion criteria, 441 patients of which 309 were from Institution 1 and 132 from Institution 2 were reviewed. Applying our exclusion criteria, a final population of 117 patients from Institution 1 (mean age 48 years, range 15-94 years), with 135 lesions (91 benign and 44 malignant), and 57 patients from Institution 2 (mean age 52 years, range 12-85 years), with 66 lesions ( 21 benign and 45 malignant), was therefore included. The flowchart of patient selection process is illustrated in Fig. 2. Age was not statistically different between training and test sets at Student's t-test ( $p=0.177)$.

Mean size of breast lesions from Institution 1 was $13 \mathrm{~mm}$ (range 4-44 mm), while mean size of breast lesions from Institution 2 was $16.37 \mathrm{~mm}$ (range 4-47 mm). Training and test sets did not differ in terms of lesion size between benign and malignant breast lesions at Mann-Whitney U test ( $p$ values 0.794 and 0.325 , respectively).

The BI-RADS assessment of the included lesions can be found in the supplementary materials as Table S1.

Fourteen BI-RADS 4 lesions were revealed as benign, while the remaining BI-RADS 4 and 2 BI-RADS 3 lesions, who showed a significant increase of lesion size at follow-up examinations, resulted in malignant pathology. Final diagnoses of histologically proven BI-RADS 3, 4, and 5 breast lesions are reported in Table 1.

\section{Feature extraction, data analysis, and feature selection}

A total of 520 features were extracted. Of these, 198 resulted unstable after ICC assessment and were discarded, with 322 features left. Then, 10 low variance parameters were also excluded as well as 278 highly intercorrelated ones, as resulted from the pairwise correlation matrix shown in Figure S1. After class balancing with SMOTE, from the remaining 34 features, RFECV identified a subset of 10 (Figure S2), including "original shape2D PerimeterSurfaceRatio"; "original shape2D Elongation"; "original glcm Autocorrelation"; "original gldm DependenceNonUniformityNormalized"; "log-sigma-1-0mm-3D glcm Imc2"; "log-sigma-2-0-mm-3D glcm Correlation"; "log-sigma-3-0-mm-3 D glrlm GrayLevelNonUniformityNormalized"; "log-sigma-4-0-mm3D glcm Imc1"; "wavelet-H glcm Imc2"; "wavelet-H glrlm GrayLevelNonUniformityNormalized." The feature selection process is summarized in Figure S3.

\section{Machine learning analysis}

The RF hyperparameters were set as follows: bootstrap $=$ true, class weight $=$ none, criterion $=$ Gini, maximum depth $=$ none, maximum features $=5$, maximum leaf nodes $=$ none, minimum impurity decrease $=0.0$, minimum impurity split $=$ none, minimum samples leaf $=1$, minimum samples split $=2$, minimum weight fraction leaf $=0.0$, number of estimators $=400$.

In the training set, RF obtained an overall mean accuracy of $82 \%$ (standard deviation, $\mathrm{SD}, \pm 6 \%$ ) and positive predictive value (PPV), sensitivity, specificity, and AUC for malignant lesions respectively of $78 \%(\mathrm{SD} \pm 5 \%$ ), $89 \%(\mathrm{SD} \pm 7 \%), 75 \%$ $(\mathrm{SD} \pm 5 \%)$, and $0.90(\mathrm{SD} \pm 0.06)$.
Fig. 2 Flowchart of the patient selection process. Pts, patients; BLs, breast lesions

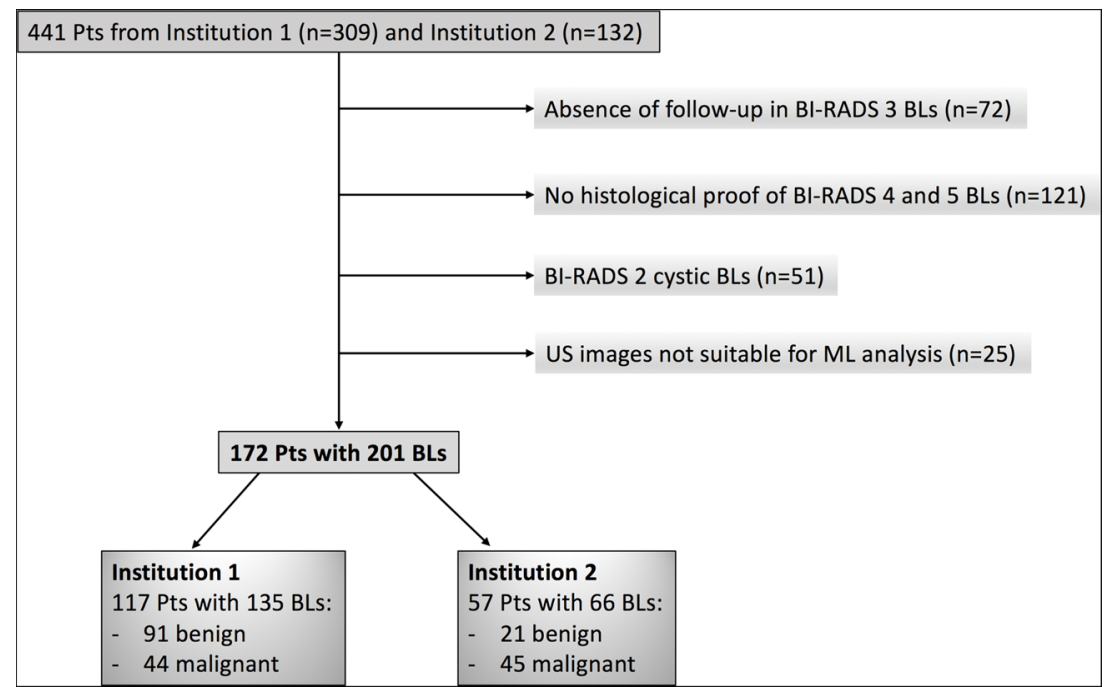


Table 1 Diagnosis of histologically proven BI-RADS 3,4 , and 5 lesions in both training and test sets

\begin{tabular}{llll}
\hline Diagnosis & Number of lesions $(\%)$ & Training $(\%)$ & Test (\%) \\
\hline Malignant lesions & & & $34(76)$ \\
Invasive ductal carcinoma & $66(75)$ & $32(73)$ & $6(13)$ \\
Invasive lobular carcinoma & $10(11)$ & $4(9)$ & $5(11)$ \\
Other* & $13(14)$ & $8(18)$ & 45 \\
Total & 89 & 44 & $8(18)$ \\
Tumor grade & & & $22(49)$ \\
G1 & $15(17)$ & $7(16)$ & $15(33)$ \\
G2 & $47(53)$ & $25(58)$ & 45 \\
G3 & $26(30)$ & $11(26)$ & $39(87)$ \\
Total & 88 & 43 & \\
Molecular subtype & & $38(88)$ & $6(13)$ \\
Luminal A and B & $77(88)$ & $2(5)$ & 45 \\
HER2+ & $2(2)$ & $3(7)$ & \\
Triple negative & $9(10)$ & 43 & $3(60)$ \\
Total & 88 & $3(33)$ & $1(20)$ \\
Benign lesions & & $3(33)$ & $1(20)$ \\
Fibroadenoma & $6(43)$ & $2(22)$ & 5 \\
Intraductal papilloma & $4(29)$ & 9 & \\
Steatonecrosis & $3(21)$ & $(12)$ & \\
Complex cyst & 14 & $93)$ & \\
Total & & & \\
\hline
\end{tabular}

*Intraductal papillary carcinoma, mucinous carcinoma, adenoid-cystic carcinoma; Hodgkin lymphoma, ductal carcinoma in situ
In the test set, ML accuracy also was $82 \%$ (95\% confidence intervals $(\mathrm{CI})=70-90 \%)$ with a PPV and negative predictive value (NPV) of $82 \%(95 \% \mathrm{CI}=74$ to $89 \%)$ and $80 \%(95 \% \mathrm{CI}$ $=56-93 \%)$, sensitivity of $93 \%(95 \% \mathrm{CI}=82-99 \%)$, and specificity of $57 \%$ (95\% CI $=34-78 \%$ ) for malignant lesions. RF's accuracy results are significantly better than the NIR $(p=0.0098)$. The AUC was $0.82(95 \% \mathrm{CI}=0.70-0.93)$ (Fig. 3). Regarding prediction and calibration loss on the test

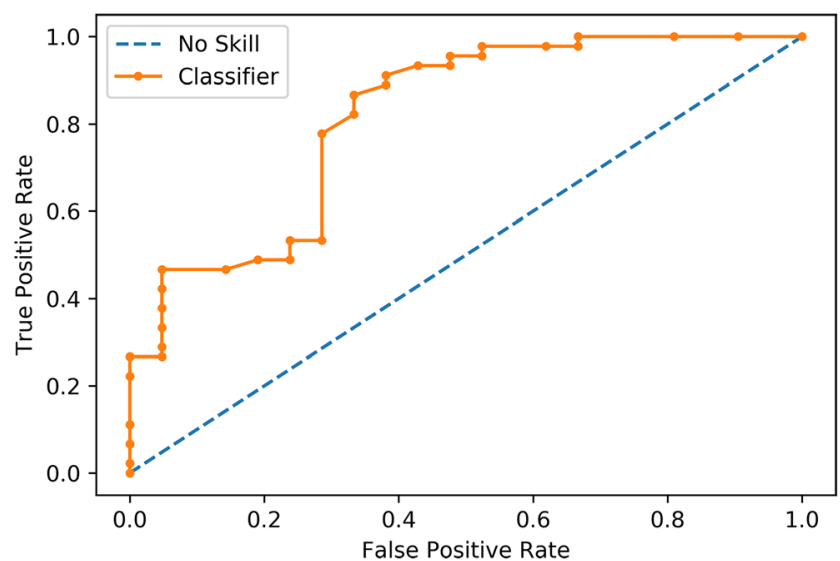

Fig. 3 Receiver operating characteristic curve of the machine learning classifier for distinguishing benign and malignant lesions in the test set set, the Brier score was 0.17 and Fig. 4 presents the calibration curve plot.

\section{Radiological evaluation}

The expert radiologist obtained an accuracy of $79.4 \%(95 \% \mathrm{CI}$ $=67-91 \%)$ on the test set, not significantly different from ML

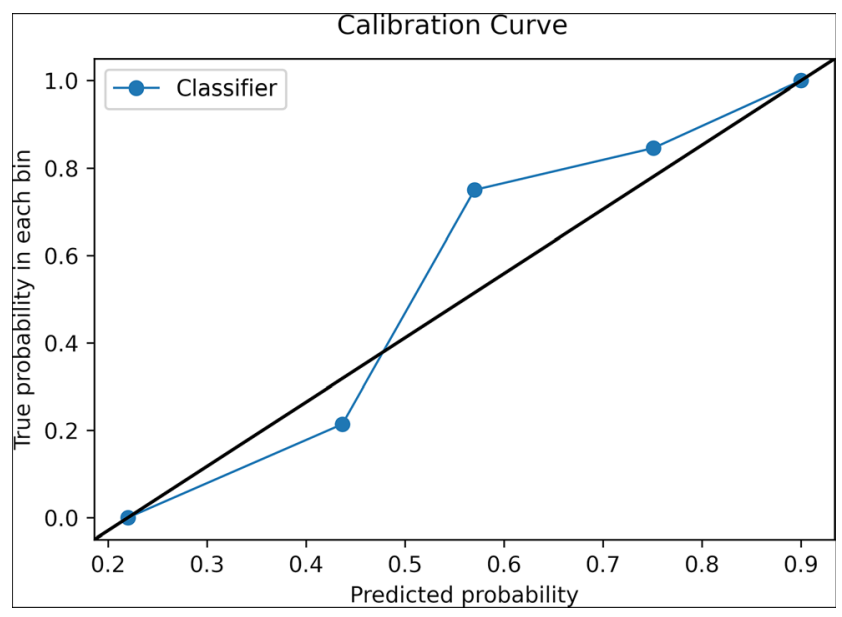

Fig. 4 Calibration curve plot of the model in the test set. Average predicted probability is represented in the $x$-axis while the proportion of malignant lesions in the $y$-axis 

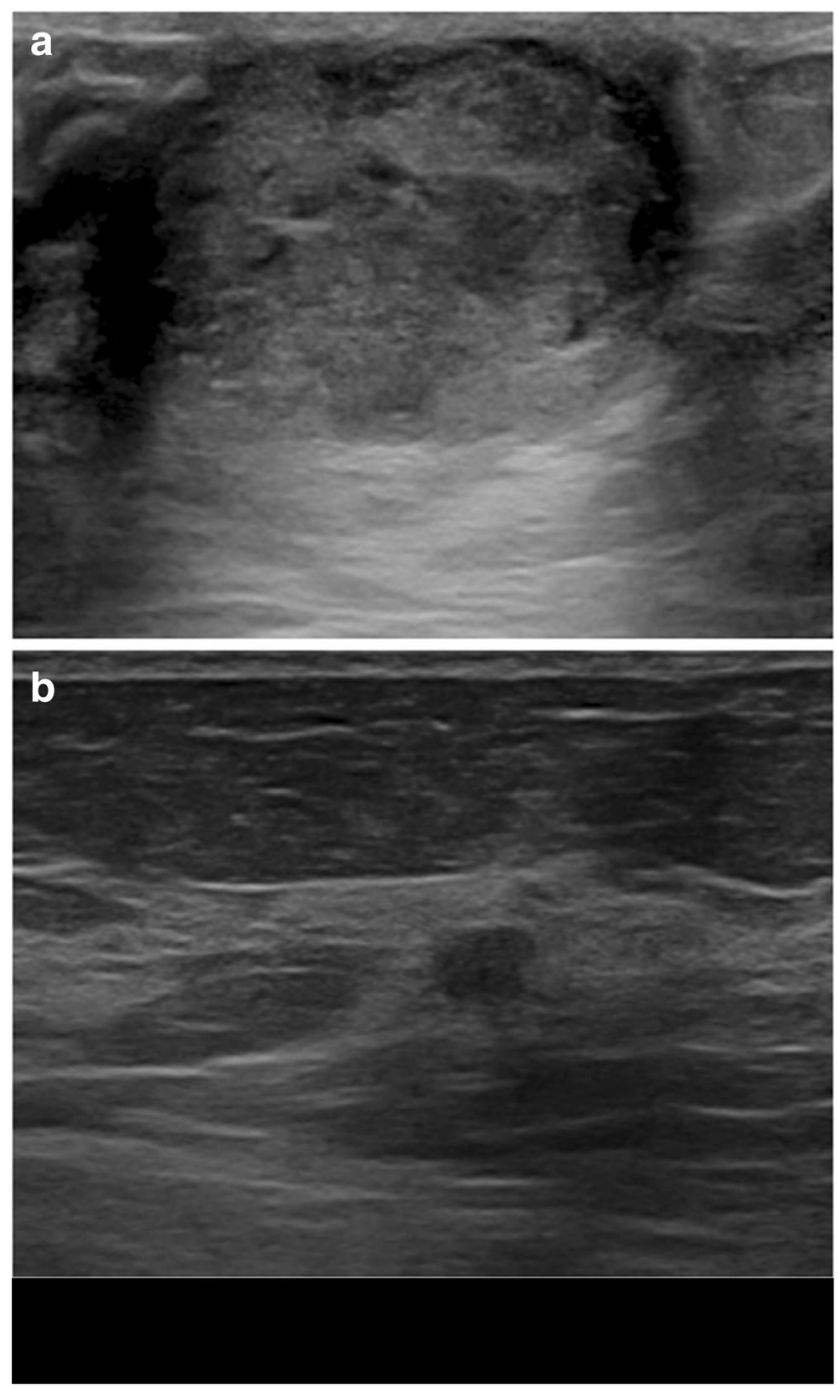

Fig. 5 B-mode US images of a benign (a) and malignant (b) breast lesion initially misclassified by the expert radiologist and correctly diagnosed with the availability of ML reading. a A case of a 13-year-old patient with a 4-cm oval breast lesion with circumscribed margins but heterogeneous echo-pattern, proved to be a sclerosing papilloma after surgical excision. b A case of a 59-year-old patient with a 5-mm oval, hypoechoic breast lesion with circumscribed margins, histologically proved as Luminal A, G1, ductal invasive carcinoma

reading at McNemar's test $(p=0.815)$. Sensitivity and specificity in identifying malignant breast lesions were $77.8 \%$ (95\% CI $=62.9$ to $88.8 \%)$ and $81 \%(95 \% \mathrm{CI}=58.1$ to 94.6\%), respectively, while PPV was $89.7 \%(95 \% \mathrm{CI}=78.1$ to $95.5 \%$ ) and NPV $63 \%$ (95\% CI $=48.7$ to $75.3 \%$ ). With the availability of ML predictions, these metrics improved as follows: accuracy $=80.2 \%(95 \% \mathrm{CI}=67-93 \%)$, sensitivity $=$ $88.9 \%(95 \% \mathrm{CI}=75.6$ to $96.3 \%)$, specificity $=71.4 \%(95 \%$ $\mathrm{CI}=47.8$ to $88.7 \%), \mathrm{PPV}=87 \%(95 \% \mathrm{CI}=77.1$ to $93 \%)$, and $\mathrm{NPV}=75 \%(95 \% \mathrm{CI}=55.7$ to $87.7 \%)$. The McNemar test comparing the $\mathrm{ML}$ and expert radiologist's readings was not significantly different $(p=0.508)$. Classification tables of the comparison between the performance of the expert radiologist and ML as well as between the expert radiologist without and with the support of ML are reported in Tables S2 and S3, respectively. Examples of cases in which the expert radiologist was aided by the ML algorithm in correctly classifying benign and malignant lesions are illustrated in Fig. 5 . Accuracy metrics of ML and expert radiologist without and with the availability of ML prediction are summarized in Table 2.

\section{Discussion}

In this study, we built a radiomic-based ML model to differentiate benign from malignant breast lesions on US. The resulting RF algorithm obtained an accuracy of $82 \%$, with high sensitivity (93\%) but low specificity (57\%) in classifying benign and malignant breast lesions. It performed significantly better than the baseline NIR $(p=0.0098)$ and showed higher accuracy compared to the expert radiologist (82\% vs 79.4\%), even if this difference did not reach statistical significance $(p=0.815)$. Even though the radiologist's accuracy increased to $80.2 \%$ with the aid of ML predictions, this difference also proved not statistically significant $(p=0.508)$.

As with all ML studies, our results should be interpreted by taking into account the data that was employed to build and test the model. We wished to focus on challenging lesions, excluding all cystic lesions which do not pose a real diagnostic challenge. This design choice is also reflected in the performance of the breast radiologist, slightly lower than what could be expected from the literature $[3,26]$. It should be acknowledged that reviewing US images is not the same as performing a complete examination, but this could not be avoided due to the study's retrospective nature. In this setting, RF outperformed both the baseline reference and the radiologist,

Table 2 Accuracy metrics (95\% confidence interval) of ML classifier and expert radiologist without and with the availability of ML reading

\begin{tabular}{|c|c|c|c|c|c|c|c|c|c|}
\hline & Accuracy & Sensitivity & Specificity & PPV & NPV & $\mathrm{TP}$ & FN & $\mathrm{TN}$ & FP \\
\hline ML classifier & $82(70-90)$ & $93(82-99)$ & $57(34-78)$ & $82(74-89)$ & $80(56-93)$ & 42 & 3 & 12 & 9 \\
\hline Expert reader & $79.4(67-91)$ & $77.8(62.9-88.8)$ & $81(58.1-94.6)$ & $89.7(78.1-95.5)$ & $63(48.7-75.3)$ & 35 & 10 & 17 & 4 \\
\hline Expert reader with ML readings & $80.2(67-93)$ & $88.9(75.6-96.3)$ & $71.4(47.8-88.7)$ & $87(77.1-93)$ & $75(55.7-87.7)$ & 40 & 5 & 15 & 6 \\
\hline
\end{tabular}

$M L$ machine learning, $P P V$ positive predictive value, $N P V$ negative predictive value. Data are expressed as percentages 
demonstrating promising performance. The improved performance of the radiologist with the aid of ML is also suggestive of its usefulness in clinical practice. Indeed, the sensitivity of the expert radiologist raised up to $88.9 \%$ vs $77.8 \%$, to the detriment of the specificity that, instead, decreased from 81 to $71 \%$. The increase in terms of sensitivity is advisable as it could reduce the possibility to miss malignant lesions. It is interesting to note that the radiologist with ML still performed worse than ML alone, probably due to lack of trust in the model's predictions.

While the model's accuracy was stable across the crossvalidation and test set assessment, in the latter we observed a reduction in specificity, compensated by increased sensitivity. It must be considered that the test set had a different proportion of malignant cases $(n=45 / 66,68 \%)$ compared to the training one $(44 / 135,33 \%)$, and overall challenging lesions, as demonstrated by the radiologist's performance.

Our findings support a possible clinical role for a US radiomic-ML tool in the characterization of benign and malignant breast lesions, in line with previous studies conducted using US radiomic features with [27-29] and without ML [12, 13, 30, 31]. For example, logistic regression models were developed using radiomic features extracted from US images to discriminate benign from malignant lesions [31], predict the presence of cancer in BIRADS 4 and 5 lesions [12], and differentiate fibroadenomas from triple negative breast cancer [13] with AUC values of $0.886,0.928$, and $0.834-0.864$, respectively. Furthermore, images obtained from automated whole breast US were used to extract texture, shape, and ellipsoid features and also analyzed using a logistic regression model, reporting an accuracy of $85 \%$ in classifying breast masses [30]. Deep learning networks were also tested to characterize benign and malignant breast lesions, with AUC values ranging from 0.80 [28] to 0.95 [29]. ML algorithms using radiomic features based on US BIRADS lexicon were also assessed by Fleury et al [32]; the Support Vector Machine resulted as the best classifier, with an AUC value of 0.84 in characterizing breast lesions. To the best of our knowledge, this is the first study assessing the usefulness of a ML classifier using shape, first order, and texture features from both original and filtered US images to classify benign and malignant breast lesions in a challenging dataset. The possibility to implement such a tool in the routine clinical practice would have tremendous implications in the management of breast lesions, considering that US is the first-level imaging modality for their assessment. In a future perspective, it would be possible to non-invasively characterize breast lesions using a widespread imaging modality, thus reducing the recourse to breast biopsy, as well as to reserve the use of second level and more expensive imaging techniques, such as MRI, to selected cases.

Limitations of our study are represented by its retrospective nature and the relatively limited patient population. However, the multicentric study design allowed to validate the AI algorithm on an external test set from a different institution, thus determining its robustness and generalizability. As stated above, the retrospective nature of the study did not allow for a standard evaluation of patients by the radiologist, who was limited to reviewing US images. Also, the final populations from the two institutions showed different proportions of malignant lesions, also understandable in light of the retrospective design of the investigation.

In conclusion, a radiomic approach paired to ML was accurate to differentiate benign from malignant BIRADS 2-5 breast lesions on US, showing a performance comparable to that of an experienced radiologist. Further studies on a larger cohort of patients and with a prospective design are necessary to confirm our promising findings.

Supplementary Information The online version contains supplementary material available at https://doi.org/10.1007/s00330-021-08009-2.

Acknowledgements Open access funding provided by Università degli Studi di Napoli Federico II within the CRUI-CARE Agreement.

Funding The authors state that this work has not received any funding.

\section{Declarations}

Guarantor The scientific guarantor of this publication is Professor Arturo Brunetti.

Conflict of interest The authors of this manuscript declare no relationships with any companies whose products or services may be related to the subject matter of the article.

Statistics and biometry No complex statistical methods were necessary for this paper.

Informed consent Written informed consent was waived by the Institutional Review Board.

Ethical approval Institutional Review Board approval was obtained.

\section{Methodology}

- Retrospective

- Observational

- Multicenter study

Open Access This article is licensed under a Creative Commons Attribution 4.0 International License, which permits use, sharing, adaptation, distribution and reproduction in any medium or format, as long as you give appropriate credit to the original author(s) and the source, provide a link to the Creative Commons licence, and indicate if changes were made. The images or other third party material in this article are included in the article's Creative Commons licence, unless indicated otherwise in a credit line to the material. If material is not included in the article's Creative Commons licence and your intended use is not permitted by statutory regulation or exceeds the permitted use, you will need to obtain 
permission directly from the copyright holder. To view a copy of this licence, visit http://creativecommons.org/licenses/by/4.0/.

\section{References}

1. Evans A, Trimboli RM, Athanasiou A et al (2018) Breast ultrasound: recommendations for information to women and referring physicians by the European Society of Breast Imaging. Insights Imaging 9:449-461. https://doi.org/10.1007/s13244-018-0636-Z

2. Mendelson EB, Böhm-Vélez M, Berg WA et al (2013) ACR BIRADS ${ }^{\circledR}$ ultrasound. In: American College of Radiology (ed) ACR BI-RADS ${ }^{\circledR}$ Atlas, Breast Imaging Reporting and Data System

3. Sood R, Rositch AF, Shakoor D et al (2019) Ultrasound for breast cancer detection globally: a systematic review and meta-analysis. J Glob Oncol:1-17. https://doi.org/10.1200/JGO.19.00127

4. Rizzo S, Botta F, Raimondi S et al (2018) Radiomics: the facts and the challenges of image analysis. Eur Radiol Exp 2:36. https://doi. org/10.1186/s41747-018-0068-z

5. van Timmeren JE, Cester D, Tanadini-Lang S, Alkadhi H, Baessler B (2020) Radiomics in medical imaging - "how-to" guide and critical reflection. Insights Imaging 11:91. https://doi.org/10.1186/ s13244-020-00887-2

6. Lambin P, Leijenaar RTH, Deist TM et al (2017) Radiomics: the bridge between medical imaging and personalized medicine. Nat Rev Clin Oncol 14:749-762. https://doi.org/10.1038/nrclinonc. 2017.141

7. Hosny A, Parmar C, Quackenbush J, Schwartz LH, Aerts HJWL (2018) Artificial intelligence in radiology. Nat Rev Cancer 18:500 510. https://doi.org/10.1038/s41568-018-0016-5

8. Choy G, Khalilzadeh O, Michalski M et al (2018) Current applications and future impact of machine learning in radiology. Radiology 288:318-328

9. Erickson BJ, Korfiatis P, Akkus Z, Kline TL (2017) Machine learning for medical imaging. Radiographics 37:505-515. https://doi. org/10.1148/rg.2017160130

10. Crivelli P, Ledda RE, Parascandolo N, Fara A, Soro D, Conti M (2018) A New challenge for radiologists: radiomics in breast cancer. Biomed Res Int 2018:1-10. https://doi.org/10.1155/2018/ 6120703

11. Tagliafico AS, Piana M, Schenone D, Lai R, Massone AM, Houssami N (2020) Overview of radiomics in breast cancer diagnosis and prognostication. Breast 49:74-80. https://doi.org/10. 1016/j.breast.2019.10.018

12. Luo WQ, Huang QX, Huang XW, Hu HT, Zeng FQ, Wang W (2019) Predicting Breast Cancer in Breast Imaging Reporting and Data System (BI-RADS) ultrasound category 4 or 5 lesions: a nomogram combining radiomics and BI-RADS. Sci Rep. https://doi. org/10.1038/s41598-019-48488-4

13. Lee SE, Han K, Kwak JY, Lee E, Kim EK (2018) Radiomics of US texture features in differential diagnosis between triple-negative breast cancer and fibroadenoma. Sci Rep 8:13546. https://doi.org/ 10.1038/s41598-018-31906-4

14. Yushkevich PA, Gao Y, Gerig G (2016) ITK-SNAP: An interactive tool for semi-automatic segmentation of multi-modality biomedical images. In: 2016 38th Annual International Conference of the IEEE Engineering in Medicine and Biology Society (EMBC). IEEE, pp 3342-3345

15. Koo TK, Li MY (2016) A guideline of selecting and reporting intraclass correlation coefficients for reliability research. J Chiropr Med 15:155-163. https://doi.org/10.1016/j.jcm.2016.02.012
16. van Griethuysen JJM, Fedorov A, Parmar C et al (2017) Computational radiomics system to decode the radiographic phenotype. Cancer Res 77:e104-e107. https://doi.org/10.1158/00085472.CAN-17-0339

17. Schwier M, van Griethuysen J, Vangel MG et al (2019) Repeatability of multiparametric prostate MRI radiomics features. Sci Rep 9:9441. https://doi.org/10.1038/s41598-019-45766-Z

18. Mlsna PA, Rodríguez JJ (2009) Gradient and Laplacian edge detection. In: The essential guide to image processing. Elsevier, pp 495-524

19. Moulin P (2009) Multiscale image decompositions and wavelets. In: The Essential guide to image processing. Elsevier, pp 123-142

20. Du R, Lee VH, Yuan H et al (2019) Radiomics model to predict early progression of nonmetastatic nasopharyngeal carcinoma after intensity modulation radiation therapy: a multicenter study. Radiol Artif Intell 1:e180075. https://doi.org/10.1148/ryai.2019180075

21. 3.6.1. RDCT (2019) A language and environment for statistical computing. R Found. Stat. Comput. 2:https://www.Rproject.org

22. Chawla NV, Bowyer KW, Hall LO, Kegelmeyer WP (2002) SMOTE: synthetic minority over-sampling technique. J Artif Intell Res 16:321-357. https://doi.org/10.1613/jair.953

23. Fernandez A, Garcia S, Herrera F, Chawla NV (2018) SMOTE for learning from imbalanced data: progress and challenges, marking the 15-year anniversary. J Artif Intell Res 61:863-905. https://doi. org/10.1613/jair.1.11192

24. Pedregosa F, Varoquaux G, Gramfort A, et al (2012) Scikit-learn: machine learning in Python

25. Cuocolo R, Caruso M, Perillo T, Ugga L, Petretta M (2020) Machine learning in oncology: a clinical appraisal. Cancer Lett 481:55-62. https://doi.org/10.1016/j.canlet.2020.03.032

26. Gharekhanloo F, Haseli MM, Torabian S (2018) Value of ultrasound in the detection of benign and malignant breast diseases: a diagnostic accuracy study. Oman Med J. https://doi.org/10.5001/ omj.2018.71

27. Becker AS, Mueller M, Stoffel E, Marcon M, Ghafoor S, Boss A (2017) Classification of breast cancer from ultrasound imaging using a generic deep learning analysis software: a pilot study. Br J Radiol 91:20170576. https://doi.org/10.1259/bjr.20170576

28. Cao Z, Duan L, Yang G, Yang G, Yue T, Chen Q (2019) An experimental study on breast lesion detection and classification from ultrasound images using deep learning architectures. BMC Med Imaging 19:51. https://doi.org/10.1186/s12880-019-0349-x

29. Qian X, Zhang B, Liu S et al (2020) A combined ultrasonic B-mode and color Doppler system for the classification of breast masses using neural network. Eur Radiol 30:3023-3033. https://doi.org/ 10.1007/s00330-019-06610-0

30. Moon WK, Shen Y-W, Huang C-S, Chiang L-R, Chang R-F (2011) Computer-aided diagnosis for the classification of breast masses in automated whole breast ultrasound images. Ultrasound Med Biol 37:539-548. https://doi.org/10.1016/j.ultrasmedbio.2011.01.006

31. Sivaramakrishna R, Powell KA, Lieber ML, Chilcote WA, Shekhar R (2002) Texture analysis of lesions in breast ultrasound images. Comput Med Imaging Graph 26:303-307. https://doi.org/10.1016/ S0895-6111(02)00027-7

32. Fleury E, Marcomini K (2019) Performance of machine learning software to classify breast lesions using BI-RADS radiomic features on ultrasound images. Eur Radiol Exp 3:34. https://doi.org/10. 1186/s41747-019-0112-7

Publisher's note Springer Nature remains neutral with regard to jurisdictional claims in published maps and institutional affiliations. 